\title{
A Redundancy Dual-backup Method of the Battery Pack for the Two-wheeled Self-balanced Vehicle
}

\author{
Yong Tao ${ }^{1, a^{*}}$, Tianmiao Wang ${ }^{1, b}$, Ye Wang ${ }^{2, c}$ and Zhongyuan Chen ${ }^{2, d}$ \\ ${ }^{1}$ Beihang University, Beijing, China \\ ${ }^{2}$ Ninebot Inc., Beijing, China

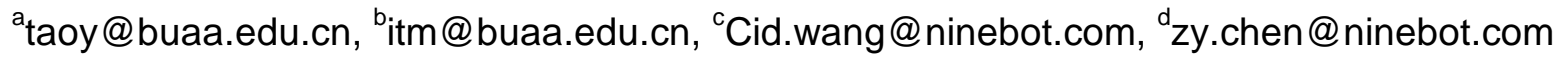

\begin{abstract}
Keywords: Dual redundancy backup, Battery pack, Self-balanced vehicle, Balance load
Abstract. In order to improve the stability and reliability of the two-wheeled self-balanced vehicle, a redundancy dual-backup method of the battery pack is proposed. The redundancy dual-backup scheme for the power supply system is presented, and the failure detection and switching method are given. In order to keep the balance of the batteries, the composed method effectively avoids the nonlinear impact and imbalance load emerges in the two groups of the battery. The experimental results show that the redundancy dual-backup method could prevent the failure of the battery and enhance the safety and reliability of the vehicle.
\end{abstract}

\section{Introduction}

Self-balance system is a combination of robot technology, intelligent control and embedded mechatronics technology. Through the detection and analysis of the system posture, the self-balance system keeps the state variables in a preset band and keep the system balance.

Relative to the conventional four wheeled vehicle, the two wheeled self-balanced electric vehicle has the advantages such as energy saving and environmental protection. The two wheeled vehicles are used in the narrow space shuttle, the exhibition inspection and other special occasions. It can be used as a research platform of the intelligent control algorithm. It is one of the research hotspots in the field of electric vehicles theory and engineering.

The self-balanced electric vehicle is arranged in two rounds, which is combined of the linear inverted pendulum and the rotary inverted pendulum. With the characteristics of flexible movement, intelligent control and environment friendly, the self-balanced electric vehicle is a typical unstable, nonlinear and strong coupling system.

An If-Then fuzzy rule base is built to cooperate with the parallel distributed compensation control of the self-balanced transporter [1]. A tilting-type balancing mobile robot platform is investigated for enhancing lateral stability [2]. A self-balanced human transportation vehicle is proposed for the teaching of feedback control concepts in undergraduate electrical, mechatronic, and mechanical engineering environments [3]. A wheeled human-conveyance vehicle with a low-level microprocessor is realized in [4], and a simple Mamdani-like fuzzy controller is given for the self-balanced vehicle control. An electric drive system for self-balanced vehicles is presented in [5].

A method for the estimation of the battery pack state of charge based on Unscented Particle Filter (UPF) algorithm is established [6]. The battery pack modeling for the analysis of battery management system of a hybrid electric vehicle is given [7].

The modeling, system design, control and implementation of two wheeler self-balanced vehicle is introduced in [8-10]. The analysis and design of a passive steering mechanism for a pedaled, self-balanced, personal mobility vehicle is proposed in [11].

As the power source of the two-wheeled vehicle, the Lithium battery is the energy storage device. It has the advantages of high working voltage, high energy density, low self-discharge rate and no memory effect. The use of lithium batteries help to reduce the volume and weight of the vehicle besides guarantee the ability of the endurance. The reliability of electric appliance directly affects the driving safety of the vehicle. If there is a battery failure in the course of the vehicle ride, the vehicle's balance system will not continue to work and it will cause casualties. 
The safe and reliable operation of the two wheeled self-balanced vehicle is key technology under different motion states such as no-load, manned, forward, backward, rotating and braking. A dual redundancy thermal backup method of the battery pack is proposed. The failure detection and switching method are presented. The experimental results verify the effectiveness of the proposed method.

\section{Introduction of the two-wheeled self-balanced Vehicle}

The dual redundancy thermal backup method is presented to improve the self-balanced vehicle safety of two kinds of components. One is the component with higher failure rate and another one is the component whose failure will affect the safety of the components. The vehicle actuator, the control and drive circuit, the battery, the gyroscope attitude and steering sensor, the signal conditioning circuit.

According to the redundancy dual-backup design, the two sets of the working system and the backup system are running at the same time. The working system and backup system monitor the working state through the digital communication mode. Once the backup system detects the system working fault, it immediately deprives the control of the system and upgrades to the working system. At the same time, it sends out alarm types to notify the user. The body structure layout of the two wheeled self-balanced vehicle is shown in Fig. 1.
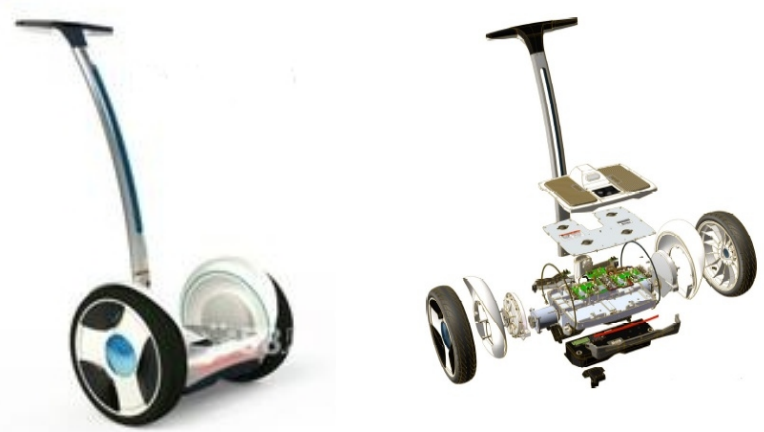

Fig. 1 the Two-wheeled Self-balanced Vehicle

\section{A Redundancy Dual-backup Method of the Battery Pack}

The whole back system of the battery pack is comprised of two batteries, two battery status monitors, MCU micro controller, two groups of controllable switch components and power system as shown in Fig. 2.

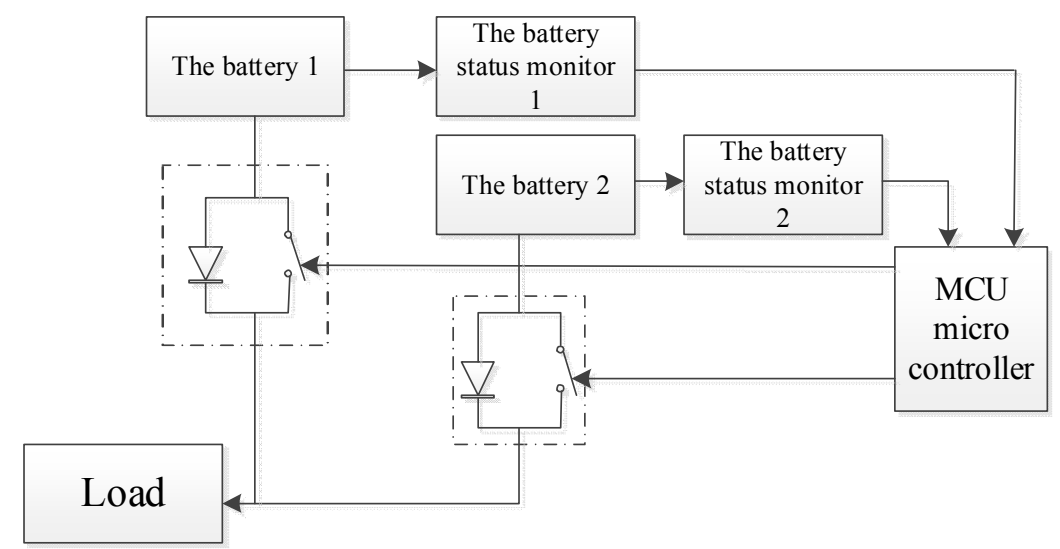

Fig. 2 Diagram of the system function module

The monitor of the battery status is used to monitor the voltage, current and capacity of the two batteries on-line. The micro controller determines the working condition of batteries. 
A forward connected diode and an equivalent normally open switch are the main components of the controllable switch module. When the switch is in a closed state, the battery will supply to the load by a diode, which ensures the micro current supply to the system in the case of vehicle standby. Because of the conduction of the diode is based on the $0.6 \mathrm{~V}$ voltage drop, the high voltage of two batteries is priority conducted, and the corresponding diode of the lower voltage battery is closed. The two batteries balance supply to the system when the system is in the standby mode.

When the vehicle is started from the standby state, firstly the micro controller monitors the working condition of two batteries. If they are working properly, the switch of the higher battery will be opened and use the higher battery priority. Otherwise the battery will be in the alarm state.

When the self-balanced vehicle is working properly, one of the switches is closed. The micro controller unit monitors the working condition of two batteries in every 10 millisecond. If the output voltage is lower or zero caused by the fault during the riding process, the corresponding switch of the fault battery should be closed and the switch of the normal battery should be opened. Since the diode is in a reverse biased state, the fault battery has no current and the vehicle ride properly to avoid the accident. If the two batteries are both normal, the battery with higher switch will be used to keep the balance of the power system.

The work principle of the switch module is shown in Fig. 3. The main components of the switch module is composed of the two high power N-channel MOS tube device used as the switch, two isolated high-speed optocouplers and the $12 \mathrm{~V}$ switch to $12 \mathrm{~V}$ isolated DC-DC module.

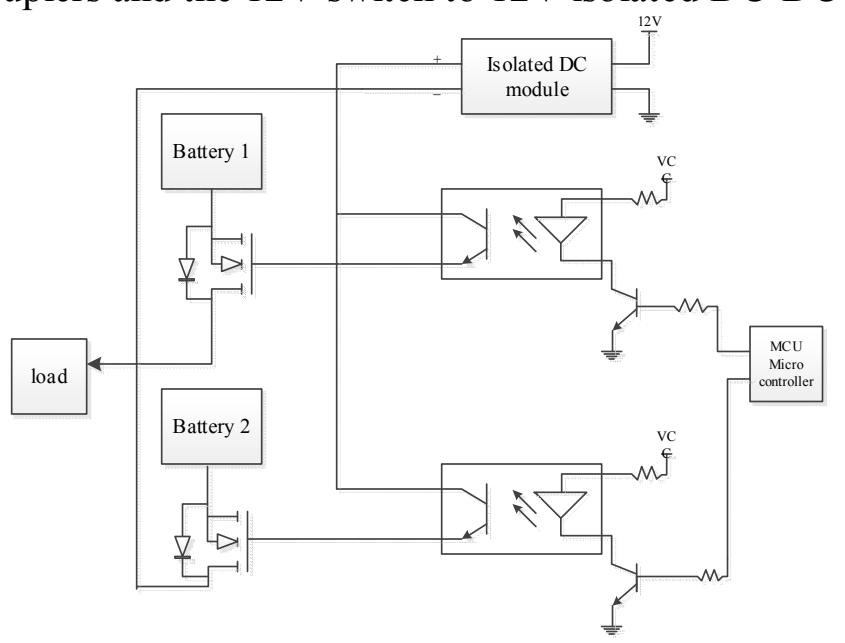

Fig. 3 the Controllable Switch Component

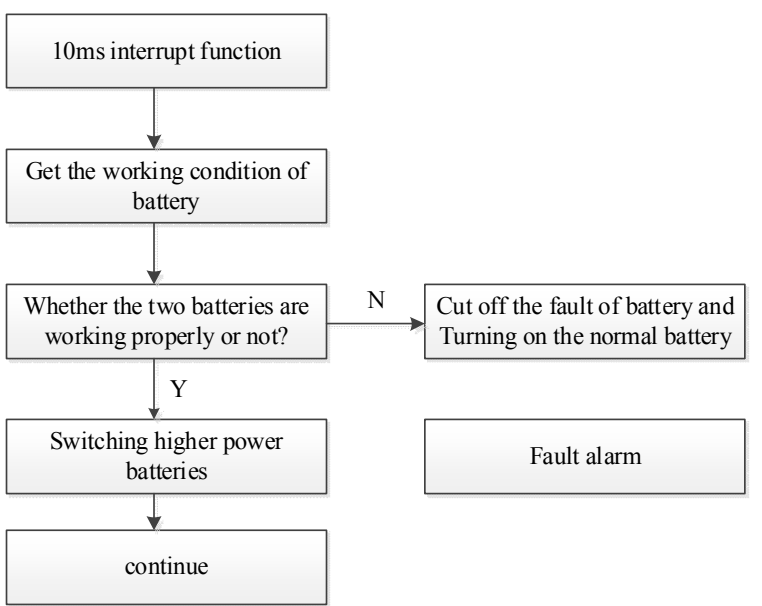

Fig. 4 Flow Chart of Control Program

The isolated DC-DC module is used to provide switching signal voltage which is higher than the $12 \mathrm{~V}$ of the power supply for the two power MOS tubes. It controls the on-off of the MOS tube by 
applied to power MOS tube gate which is added by isolated optocoulpers. The control signal of the optocoupler is given by the micro controller. The flow chart of the control program is shown in Fig. 4 .

\section{Conclusions}

For the safety of the two-wheeled self-balanced vehicle, a redundancy dual-backup method of the battery pack is proposed. When one of battery packs suffers a breakdown, it will switch to another normal battery to prevent the occurrence of accidents. There is a balance mechanism of the two battery packs, which ensures the imbalance load of battery in a long time.

\section{References}

[1] Huang, C.-H, Wang, W.-J, Chiu, C.-H. Velocity control realisation for a self-balancing transporter[J]. IET Control Theory Applications, 2011, 5(13):1551-1560.

[2] Kwon S J, Kim S, Yu J. Tilting-Type Balancing Mobile Robot Platform for Enhancing Lateral Stability[J]. Mechatronics IEEE/ASME Transactions on, 2015, 20:1470-1481.

[3] Lin S C, Tsai C C. Development of a Self-Balancing Human Transportation Vehicle for the Teaching of Feedback Control[J]. IEEE Transactions on Education, 2009, 52(1):157 - 168.

[4] Chiu C H, Chang C C. Design and Development of Mamdani-Like Fuzzy Control Algorithm for a Wheeled Human-Conveyance Vehicle Control[J]. Industrial Electronics IEEE Transactions on, 2012, 59(12):4774-4783.

[5] Hofer K. Electric drive system for self-balanced vehicles[C]// Industry Applications (INDUSCON), 2010 9th IEEE/IAS International Conference on. IEEE, 2010:1-4.

[6] Zhong L, Zhang C, He Y, et al. A method for the estimation of the battery pack state of charge based on in-pack cells uniformity analysis[J]. Applied Energy, 2014, 113(1):558-564.

[7] Sen C, Kar N C. Battery Pack Modeling For The Analysis Of Battery Management System Of A Hybrid Electric Vehicle[C]// Vehicle Power and Propulsion Conference, 2009. VPPC '09. IEEE. 2009:207-212.

[8] Rumi S M I, Hossain M F, Shanamul Islam I S M, et al. System design of two wheeler self-balanced vehicle[C]// Mecatronics (MECATRONICS), 2014 10th France-Japan/ 8th Europe-Asia Congress on. IEEE, 2014:331-336.

[9] Huang C F, Yeh T J. Modeling, control and implementation of a pedaled, self-balanced unicycle[C]// System Integration (SII), 2011 IEEE/SICE International Symposium on. IEEE, 2011:1323 - 1328 .

[10] Huang C F, Yeh T J. Modeling and control of a pedaled, self-balanced unicycle[C]// Advanced Intelligent Mechatronics (AIM), 2012 IEEE/ASME International Conference on. IEEE, 2012:965-970.

[11] Hong J H, Huang C F, Lu K Y, et al. Analysis and design of a passive steering mechanism for a pedaled, self-balanced, personal mobility vehicle[C]// System Integration (SII), 2013 IEEE/SICE International Symposium on. IEEE, 2013:179-184. 